\title{
PSEUDOSPIN MODELS OF SUPERCONDUCTORS WITH VERY SHORT COHERENCE LENGTH
}

\author{
S. RoBASZKIEWICZ \\ Institute of Physics, A. Mickiewicz University, Umultowska 85, 61-614 Poznań, Poland
}

We discuss a few models developed for description of superconductivity with very short coherence length and point out their formal equivalence to specific spin models, such as an anisotropic Heisenberg model $(s=1 / 2)$ with two- and four-spin exchange coupling and with fixed magnetization in the $z$-direction, an XY-Z model with single ion anisotropy and a Kondo-lattice model with anisotropic on-site exchange. New results concerning the phase diagram of the Hubbard model with strong attraction, the electromagnetic properties of systems with the first order superconducting transition and a possibility of local charge moment compensation in systems of coexisting bound pairs and itinerant electrons are presented.

PACS numbers: 74.20.-z, 05.30.Jp, 71.28.+d

\section{Introduction}

Recently, there has been much interest in superconductivity with very short coherence length due to its possible relevance to high-temperature superconductors (the cuprates, doped bismuthates and fullerenes) and also to the several other exotic superconducting materials [1-5]. All these materials are extreme type-II superconductors and they generally exhibit low carrier density, a small value of the Fermi energy $\left(E_{\mathrm{F}} \leq 0.1-0.3 \mathrm{eV}\right)$, a short coherence length $\xi_{0}$ and $\xi_{0} k_{\mathrm{F}} \simeq 1-10$. Thus, the size of a pair can be of the order of the interparticle distance (or even a lattice constant). Moreover, for many of these materials, there are several universal trends in the $T_{\mathrm{c}}$ versus condensate density dependence, the $T_{\mathrm{c}}$ dependence of the pressure and the isotope effect coefficient and in the temperature dependence of the penetration depth $[4,5]$.

These features strongly support the models with short-range, nonretarded attraction [see Ref. [1] for a review]. One of the simplest effective models of this type is the extended Hubbard model with an on-site attractive interaction

$$
\widehat{\Pi}=\sum_{i j \sigma} t_{i j} c_{i \sigma}^{+} c_{j \sigma}+U \sum_{i} n_{i \uparrow} n_{i \downarrow}+\frac{1}{2} \sum_{i j} W_{i j} n_{i} n_{j}-\mu \sum_{i} n_{i},
$$

where $n_{i}=n_{i \uparrow}+n_{i, \downarrow}, n_{i \sigma}=c_{i \sigma}^{+} c_{i \sigma}, t_{i j}$ denotes the transfer integral, $U$ is the on-site attractive $(U<0)$ interaction and $W_{i j}$ is the intersite density-density interaction 
between sites $i$ and $j$. The quantity $\mu$ is the chemical potential depending on the number of electrons per site

$$
n=\frac{1}{N} \sum_{i}\left\langle n_{i}\right\rangle, \quad n \in[0,2] .
$$

The parameters of (1.1) can be treated as the effective ones and are assumed to include all the possible contributions and renormalizations like those coming from the strong electron-phonon coupling or from the coupling between electrons and other electronic subsystems in solid or chemical complexes. The model (1.1) has been extensively studied for the last few years and a great deal of its properties have been established (at least in extreme limits). With increasing $|U| / t$ the system exhibits a transition from the weak coupling superconductivity of (predominantly) BCS-type to the strong coupling regime of tightly bound on-site pairs where the superconductivity results from the condensation of hard-core charged bosons (i.e. the local pairs) into a superfluid state. For a detail review of the model including thermodynamics, electromagnetic properties and critical behavior we refer the reader to Refs. [1-3].

Here, in Sec. 2 we will concentrate on the strong attraction limit and summarize our new results concerning this case. Next, in Sec. 3, we introduce a model of local pairs with finite binding energy and analyse its unique thermodynamic and electromagnetic properties. Finally, in Sec. 4, we present the model of coexisting bound pairs and itinerant electrons showing its formal equivalence with a Kondo-lattice model with anisotropic on-site exchange and discuss the CDW orderings and a possibility of local charge moment compensation in such system.

\section{Pseudospin models for strong attraction}

In the strong attraction limit, $|U| \gg t, W$, a large gap of order $|U|$, exists in the single-electron excitation spectrum for any $n$, which is equivalent to the statement that the Fermi level is pinned for $U<0$, close to its value at $n=1(\mu=$ $\left.-|U| / 2+W_{0}\right)$. Due to that fact, a standard perturbation theory for degenerate systems $[6,7]$ can be applied for the model (1.1) to derive effective Hamiltonians valid for any band-filling.

To the second order in $t_{i j} /|U|$ one obtains [8]

$$
\bar{H}=-\sum_{i j} J_{i j} \rho_{i}^{+} \rho_{j}^{-}+\sum_{i j} K_{i j} \rho_{i}^{z} \rho_{j}^{z}-\bar{\mu} \sum_{i}\left(2 \rho_{i}^{z}+1\right)+\text { const }
$$

where

$$
\begin{aligned}
& n=\frac{1}{N} \sum_{i}\left\langle 2 \rho_{i}^{z}+1\right\rangle, \\
& J_{i j}=\frac{2 t_{i j}^{2}}{|U|}, \quad K_{i j}=J_{i j}+2 W_{i j}, \quad \bar{\mu}=\mu+\frac{1}{2}|U|-\sum_{j} W_{i j} .
\end{aligned}
$$

The charge operators are

$$
\rho_{i}^{+}=\left(\rho_{i}^{-}\right)^{+}=c_{i \uparrow}^{+} c_{i \downarrow}^{+}, \quad \rho_{i}^{z}=\frac{1}{2}\left(n_{i}-1\right)
$$


and, in the subspace excluding single occupancy of sites, they satisfy the commutation rules of $s=1 / 2$ operators.

The effective pseudospin Hamiltonian (2.1) has the form of an anisotropic Heisenberg model, where the chemical potential $\bar{\mu}$ plays the role of an external field along the $z$-direction, such that the magnetization has a fixed value $(n-1) / 2$. Notice that since $\rho_{i}^{ \pm}$operators are paulions the system can be equally well considered as a charged hard-core Bose gas on a lattice [1-3] (on-site pair = hard-core boson with a charge $2 e$ ). To ensure a fixed density $n$ of electrons, the calculations on Eq: (2.1) have to be performed at constant magnetization instead of a constant field. Other differences from the standard magnetic problems concern a distance dependence of interaction parameters. In magnetic systems the interactions $J_{i j}$ and $K_{i j}$ usually have the same distance dependence and, except RKKY, they are short-range. In the present problem the kinetic charge exchange $J_{i j}$ is short-range, however $K_{i j}$ contains also the Coulomb part which can have a long-range character. Thus, for anisotropic lattices, $J_{i j}$ can be strongly unidimensional or bidimensional, whereas $K_{i j}$ will remain almost isotropic. The correspondence between electronic orderings of local pairs and types of pseudospin orderings is the following: singlet superconductivity (SS) - XY order, charge ordering (CDW) - antiferromagnetic (AF) or more complicated order along the $z$ axis, mixed CDW-SS phase (M) - intermediate state, condensed phase of electron droplets (D) - droplets of ferromagnetic order, CDW-SS phase separation (PS) - AF-XY phase separation.

For $W_{i j}$ repulsive and restricted to nearest neighbors (nn) the ground state of the system consists of the CDW phase for $n=1$, the $\mathrm{M}$ phase for $1>n>n_{\mathrm{c}}$ and SS phase for $0<n<n_{\mathrm{c}}$. The value of $n_{\mathrm{c}}$ decreases with growing $K / J$ and depends on the lattice structure [9]. Inclusion of the next $n n$ attractive interaction $W_{2}<0$ can stabilize, for $1>n>n_{\mathrm{c}}$, the PS state. Within the mean field approximation (MFA)

$$
n_{\mathrm{c}}=1-\left(\frac{K_{Q}+J_{0}}{K_{Q}+J_{Q}}\right)^{1 / 2}, \quad K_{Q}=K_{q=Q}, \quad \text { etc. }
$$

In the PS state the system breaks into coexisting domains of SS $\left(n<n_{\mathrm{c}}\right)$ and CDW $(n=1)$ phases. The sizes of the domains will be determined by long-range Coulomb repulsion. When the SS domains do not percolate one should observe a partial Meissner effect but without a zero resistance.

The Hamiltonian (2.1) includes only $t^{2} /|U|$ terms in $t /|U|$ expansion and for better description of the strong attraction limit of the model (1.1) it can be very important to evaluate the effects of higher order terms. Assuming the hopping integral $t_{i j}$ restricted to nearest neighbors $(\mathrm{nn})$ and $W_{i j}=0$ we have extended the perturbation expansion in $t /|U|$ for the attractive Hubbard model up to the fifth order. As a result one obtains the following effective Hamiltonian valid for arbitrary $n$ :

$$
\widehat{H}_{\mathrm{eff}}=\bar{\mu} \sum_{i}\left(2 \rho_{i}^{z}+1\right)+\widehat{H}_{2}+\widehat{H}_{4}^{\mathrm{nn}}+\widehat{H}_{4}^{\mathrm{nnn}}+\widehat{H}_{4}^{\mathrm{a}},
$$

where

$$
\widehat{H}_{2}=+\sum_{i<j} \frac{t_{i j}^{2}}{|U|}\left(A_{i j}-1\right)
$$




$$
\begin{aligned}
\hat{H}_{4}^{\mathrm{nn}} & =-\sum_{i<j} \frac{4 t_{i j}^{4}}{|U|^{3}}\left(A_{i j}-1\right), \\
\hat{H}_{4}^{\mathrm{nnn}} & =+\sum_{i<k} \frac{t_{i j}^{2} t_{j k}^{2}}{|U|^{3}}\left(B_{i k}-1\right), \\
\hat{H}_{4}^{\mathrm{q}} & =\sum_{i<j<l, i<k, k \neq j, l} \frac{t_{i j} t_{j k} t_{k l} t_{l i}}{|U|^{3}}\left[5 A_{i j} A_{k l}+5 A_{j k} A_{i l}-5 B_{i k} B_{j l}-\right. \\
& \left.-\left(A_{i j}+A_{j k}+A_{k l}+A_{i i}+B_{i k}+B_{j l}\right)+1\right] . \\
A_{i j} & =4\left(-\rho_{i}^{+} \rho_{j}^{-}+\rho_{i}^{z} \rho_{j}^{z}\right), \quad B_{i k}=4\left(\rho_{i}^{+} \rho_{k}^{-}+\rho_{i}^{z} \rho_{k}^{z}\right), \\
\bar{\mu}=\mu & =\frac{|U|}{2} .
\end{aligned}
$$

$\widehat{H}_{4}$ involves sums only over sets of sites connected by nn links. The diagrams corresponding to particular terms in (2.5) are shown in Fig. 1.

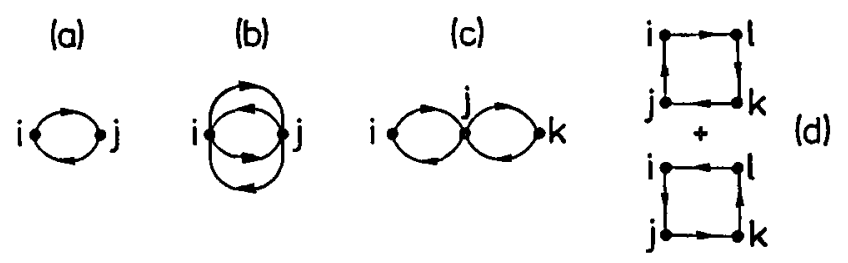

Fig. 1. The diagrams corresponding to the particular terms in the effective Hamiltonian (2.5). (a), (b), (c) and (d) corresponds to $H_{2}, H_{4}^{\text {nn }}, H_{4}^{\text {nnn }}$ and $H_{4}^{\text {D }}$, respectively.

As we see, with increasing $t /|U|$, the range of effective interactions between pairs expands and the multisites effective couplings can develop and become important. Hamiltonian (2.5) is again a pseudospin model and it has the form of a generalized Heisenberg model in external field with two-spin and four-spin exchange couplings and fixed magnetization.

The fourth order terms include: (i) the nn two-site coupling $H_{4}^{\mathrm{nn}}$ which renormalizes (reduces) the $t_{i j}^{2} / U$ term, (ii) the next nn two-site terms $H_{4}^{\text {nnn }}$, (iii) the four-site term $H_{4}^{\square}$, analogous to the cyclic four-spin exchange terms in solid ${ }^{3} \mathrm{He}$ and in magnets. Obviously, the multisite term contributes in 2 and 3 dimensions only, and in the case of a linear chain the effective Hamiltonian reduces to

$$
\begin{aligned}
\widehat{H}= & \bar{\mu} \sum_{i}\left(2 \rho_{i}^{z}+1\right)+J_{1} \sum_{i}\left\{4\left[-\frac{1}{2}\left(\rho_{i}^{+} \rho_{i+1}^{-}+\text {h.c. }\right)+\rho_{i}^{z} \rho_{i+1}^{z}\right]-1\right\} \\
& +J_{2} \sum_{i}\left\{4\left[\frac{1}{2}\left(\rho_{i}^{+} \rho_{i+2}^{-}+\text {h.c. }\right)+\rho_{i}^{z} \rho_{i+2}^{z}\right]-1\right\},
\end{aligned}
$$

where $J_{1}=t^{2} /|U|-4 t^{4} /|U|^{3}, J_{2}=t^{4} /|U|^{3}$. 
For $n=1$, by making use of the attraction-repulsion canonical transformation $[1,8]$ for the Hamiltonian (2.5) one can derive the effective Hamiltonian for the half-filled repulsive Hubbard model. The result is

$$
\begin{aligned}
& \hat{H}=\sum_{i<j} \frac{t_{i j}^{2}}{U} x_{i j}-\sum_{i<j} \frac{4 t_{i j}^{4}}{U^{3}} x_{i j}+\sum_{i<k} \frac{t_{i j}^{2} t_{j k}^{2}}{U^{3}} x_{i k}+\sum_{i<j<l, i<k, k \neq j, l} \frac{t_{i j} t_{j k} t_{k l} t_{l i}}{U^{3}} \\
& \times\left(5 x_{i j} x_{k l}+5 x_{j k} x_{i l}-5 x_{i k} x_{j l}-x_{i j}-x_{j k}-x_{k l}-x_{l i}-x_{i k}-x_{j l}\right)+\text { const }
\end{aligned}
$$

where $x_{i j}=4 \sigma_{i} \cdot \sigma_{j}$ and $\left\{\sigma_{i}\right\}$ are $s=1 / 2$ operators. It agrees with the expressions obtained directly for the repulsive case by Takahashi [7] and MacDonald et al: [10].

Our effective Hamiltonians treat only $2^{N}$ states of the starting model. The other electronic excited states have higher energy $(\sim|U|)$ and their contributions to thermodynamic quantities are of the order of $\exp \left(-|U| / k_{\mathrm{B}} T\right)$. Thus the models are applicable at $k_{\mathrm{B}} T \ll|U|$. The Hamiltonian (2.5) provides a good starting point for a further analysis of the strong attraction limit of the Hubbard model at arbitrary electron concentrations and well-founded methods of quantum theory of magnetism can be used to study its thermodynamical properties.

As the first step in the investigation we have applied the mean field approximation (MFA) to Eq. (2.5), as it usually gives correct qualitative information. The phase diagram obtained in this way for sc lattice and two fixed values of $n$ is shown in Fig. 2. As we see the fourth order terms reduce $T_{\mathrm{c}}$. With decreasing $|U|$

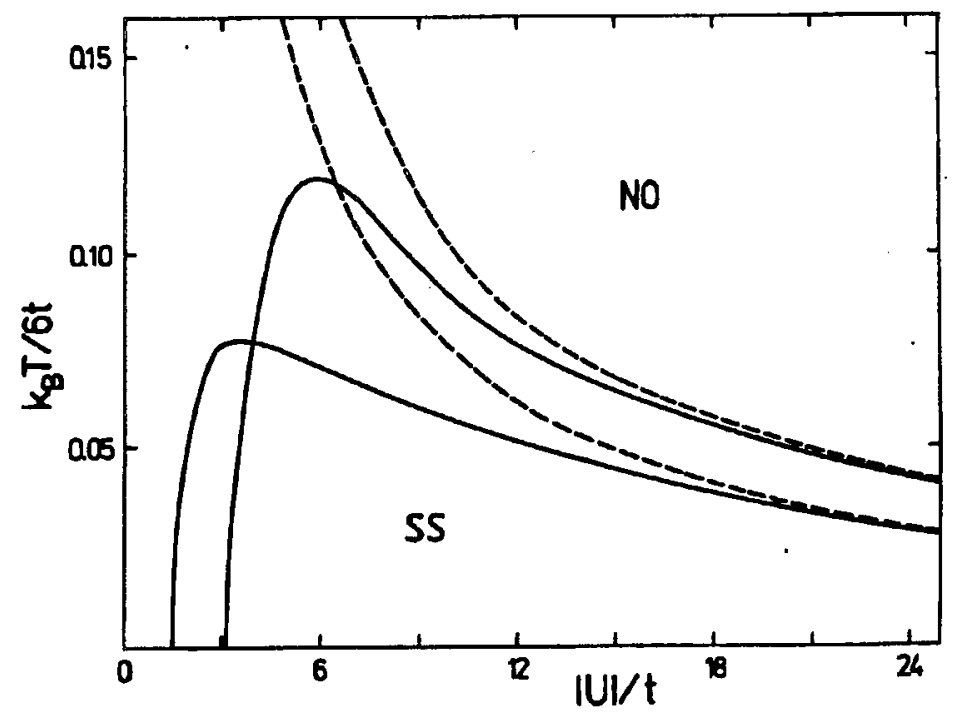

Fig. 2. Phase diagram of the attractive Hubbard model derived using the effective Hamiltonian (2.5) treated within MFA, for $n=1$ (upper solid line) and $n=0.2$ (lower solid line); sc lattice. Dashed lines denote the results obtained if (2.5) is restricted to $H_{2}$ term. 


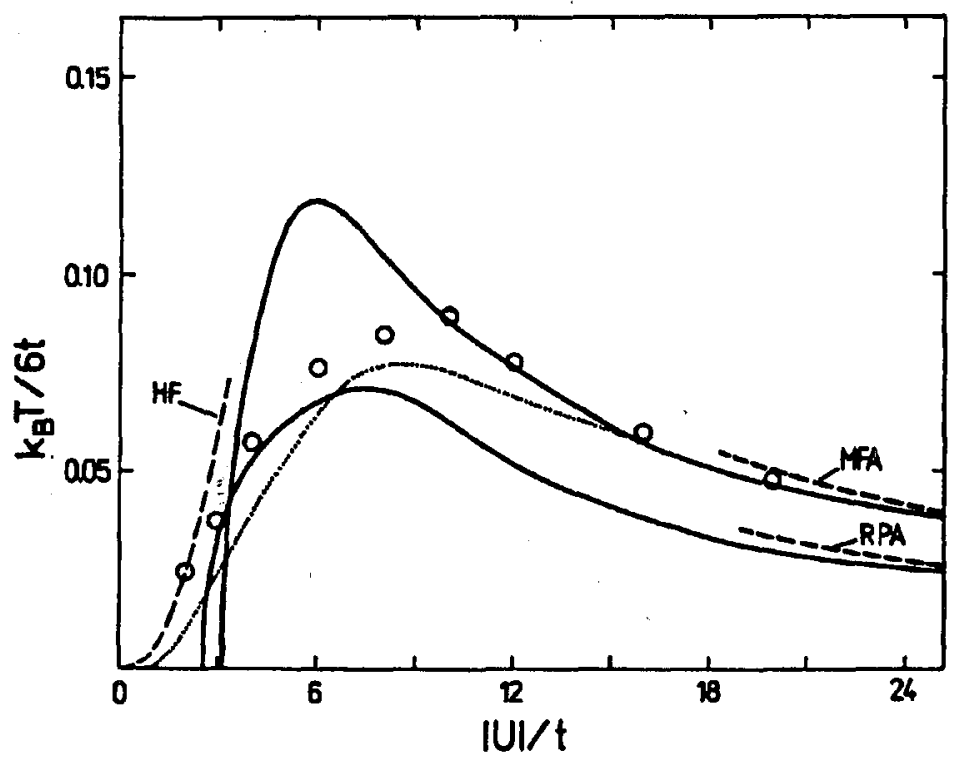

Fig. 3. The plots of $T_{c}$ vs. $|U| / t$ for the half-filled attractive Hubbard model obtained using (2.5) treated within RPA (lower solid line) and within MFA (upper solid line). Open circles and dotted line denote the results of the Monte-Carlo simulation [12] and the Gutzwiller-type variational approach [13], respectively. Dashed lines denote the RPA and MFA results obtained if (2.5) is restricted to $H_{2}$ term and the $H-F$ result.

the $T_{\mathrm{c}}$ exhibits a round maximum at a definite value of $|U| / t$ dependent on $n$ and goes to zero at some lower value of $|U| / t$. With decreasing $n$ both the maximum of $T_{\mathrm{c}}$ and the $T_{\mathrm{c}}=0$ point move towards smaller values of $|U| / t$.

For $n=1$ we have also calculated the SS critical temperature using the self-consistent random phase approximation (RPA) approach with Callen-type decoupling [11]. Figure 3 presents the RPA result and compares it with the MFA prediction and with the results obtained for the half-filled Hubbard model by applying the Monte Carlo simulations [12] and the Gutzwiller-type variational approach of Hasegawa [13]. The $T_{\mathrm{c}}$ maximum is shifted towards higher $|U| / t$ and the $T_{\mathrm{c}}=0$ point towards lower $|U| / t$ with respect to the MFA prediction. A comparison with the results of Monte-Carlo simulations and other approaches shows that the perturbative effective Hamiltonian (2.5) works quite well probably up to the maximum $T_{\mathrm{c}}$. For lower values of $|U| / t$ the expansion becomes insufficient and overestimates the effects suppressing $T_{\mathrm{c}}$.

Our results suggest that low-temperature thermodynamics of the attractive Hubbard model in the crossover regime (up to the $T_{\mathrm{c}}$ maximum ?) is governed by the effective interactions between bound pairs rather than by the pair breaking excitations. 


\section{A system of local pairs with a finite binding energy}

Let us now consider a simple model with local electron pairing taking into account the effects of finite pair binding energy $E_{\mathrm{b}}$.

$$
\begin{aligned}
\hat{H}= & \left.-\frac{1}{2} \sum_{i \neq j} J_{i j} c_{i \uparrow}^{+} c_{i \downarrow}^{+} c_{j \downarrow} c_{j \uparrow}+\text { h.c. }\right)+U \sum_{i} n_{i \uparrow} n_{i \downarrow} \\
& +\frac{1}{2} \sum_{i j \sigma \sigma^{\prime}} W_{i j} n_{i \sigma} n_{j \sigma^{\prime}}-\mu \sum_{i \sigma} n_{i \sigma} .
\end{aligned}
$$

The Hamiltonian consists of the intersite charge-exchange term $J_{i j}$, determining the pair mobility and responsible for superconducting order in the system, the effective on-site interaction $U$, which contributes to $E_{\mathrm{b}}$ by reducing $(U>0)$ or enhancing $(U<0)$ its value, and the intersite density interaction $W_{i j}$. The model corresponds to the situation when the single particle mobility is much smaller than the pair mobility and can be neglected. Our preliminary analysis indicates that Eq. (3.1) can also provide a reliable approximate description of an local pair (LP) subsystem in the model of coexisting LPs and itinerant electrons (see Sec. 4), where the effective $J_{i j}$ is generated by the intersubsystem coupling $[1,2]$.

Within the subspace excluding single occupancy of sites $(U \rightarrow-\infty)$ the model is formally reduced to the standard case of hard core bosons on a lattice (Eq. (2.1)). For arbitrary $U$ the system may be represented by a special anisotropic Heisenberg model with single ion anisotropy. Introducing the pseudospin operators

$$
\begin{aligned}
& S_{i}^{z}=\frac{1}{2}\left(n_{i \uparrow}+n_{i \downarrow}-1\right), \\
& S_{i}^{+}=c_{i \uparrow}^{+} c_{i \downarrow}^{+}=\left(S_{i}^{-}\right)^{+},
\end{aligned}
$$

Eq. (3.1) takes a form

$$
\begin{aligned}
\hat{H}= & -\frac{1}{2} \sum_{i \neq j}\left(J_{i j} S_{i}^{+} S_{j}^{-}+\text {h.c. }\right)+2 U \sum_{i}\left(S_{i}^{z}\right)^{2}+2 \sum_{i \neq j} W_{i j} S_{i}^{z} S_{j}^{z} \\
& -B \sum_{i} S_{i}^{z}+N\left(\frac{1}{2} W_{0}-\mu\right),
\end{aligned}
$$

where $B_{0}=2\left(\mu-W_{0}-U / 2\right), W_{0}=W_{q=0} .\left\{S_{i}^{\alpha}\right\}$ satisfy the usual angular momentum algebra, $\left\{S_{i}^{z}\right\}$ takes on four values $(0,0, \pm 1 / 2)$, as for $s=1$ model with doubly degenerated 0 value, whereas $\left(S_{i}^{+}\right)^{2}=\left(S_{i}^{-}\right)^{2}=0$, as in the $s=1 / 2$ system. Again XY order and AF order along $z$ axis of pseudospins correspond to SS and CDW orderings, respectively, and the magnetic field $B(\sim \mu)$ is determined by a fixed value of magnetization: $1 / N \sum_{i}\left\langle S_{i}^{z}\right\rangle=(n-1) / 2$.

\subsection{Charge orderings for $J_{i j}=0$}

Extensive analysis of charge orderings in the model (3.1) with $J=0$ has been performed by us in Refs. [14-16], within a framework of a variational approach, and most recently in Ref [17] using the cluster approximation (of BPW-type). In the case of nn repulsion $(W>0)$ the system can exhibit two types of CDW orderings: $\mathrm{C} 1$, involving the on-site pairing of electrons $(n<1)$ or holes $(n>1)$ 
(which corresponds to the alternating valence: $K^{m}, K^{m+2}$ ), and C2, which is the ordering without on-site pairs $\left(K^{m}, K^{m+1}\right)$. C1 can be a stable phase only if $U /(z W)<1$ and C2 if $U>0$. For $0<U /(z W)<1$ both types of order can be realized, depending on $n$. At $T=0$ there are the percolation thresholds for long range orderings. In particular, for sc lattice the BPW calculation predicts [17] that CDW is restricted to $0.33<n<1.77$, for $U<0$, and to $0.165<n<0.835$, $1.165<n<1.835$, for $U /(z W) \gg 1$. At finite temperature the system exhibits very rich multicritical behavior and the phase diagrams can include a tricritical line, a critical-end-point and a line of isolated critical points [14-17].

\subsection{Properties of the superconducting phase}

In the analysis of this problem we have assumed $W_{i j}=0$ in Eq. (3.1) and adopted a variational approach which treats the on-site interaction exactly and the pair hopping term within MFA (site-dependent). After diagonalization of a trial Hamiltonian, the free energy functional including the orbital and paramagnetic effects of magnetic field is obtained as [17]

$$
\begin{aligned}
F_{0}= & -\frac{1}{\beta} \sum_{m} \ln 2 Z_{m}+\frac{1}{2} \sum_{m m^{\prime}}\left(J_{m m^{\prime}} \psi_{m}^{+} \psi_{m^{\prime}}^{-} \mathrm{e}^{\left.\mathrm{i} \phi_{m m^{\prime}}+\text { h.c. }\right)}\right. \\
& +\bar{\mu}(n-1) N+\frac{n}{2} N U
\end{aligned}
$$

where

$$
\begin{aligned}
& Z_{m}=\exp \left(\beta \frac{U}{2}\right) \cosh \left(\beta H_{\mathrm{p}}\right)+\cosh \left(\beta \Delta_{m}\right), \\
& \Delta_{m}=\left(\bar{\mu}^{2}+\left|\Omega_{m}^{+}\right|^{2}\right)^{1 / 2}, \\
& \Omega_{m}^{ \pm}=\sum_{m^{\prime}} J_{m m^{\prime}} \psi_{m^{\prime}}^{ \pm} \exp \left(\mp \mathrm{i} \phi_{m m^{\prime}}\right), \quad \phi_{m m^{\prime}}=\frac{2 e}{\hbar c} \int_{r_{m}}^{r_{m^{\prime}}} \boldsymbol{A} \cdot \mathrm{d} r .
\end{aligned}
$$

$\psi_{m}^{+}=\left\langle c_{m \uparrow}^{+} c_{m \downarrow}^{+}\right\rangle=\left(\psi_{m}^{-}\right)^{+}, H_{\mathrm{p}}=g \mu_{\mathrm{B}} H, \boldsymbol{A}$ is the vector potential, $r_{m}$ is the coordinate of $m$ site and $\bar{\mu}=\mu-U / 2$.

From $\partial F_{0} / \partial \psi_{m}^{ \pm}=0$ and $\partial F_{0} / \partial \bar{\mu}=0$ one gets a set of equations determining the superconducting order parameter $\psi_{m}^{ \pm}$and the chemical potential $\bar{\mu}$

$$
\begin{aligned}
\psi_{m}^{ \pm} & =\frac{\Omega_{m}^{ \pm}}{2 \Delta_{m}} \frac{\sinh \left(\beta \Delta_{m}\right)}{\exp (\beta U / 2) \cosh \left(\beta H_{\mathrm{p}}\right)+\cosh \left(\beta \Delta_{m}\right)}, \\
\frac{n-1}{2} & =\frac{1}{N} \sum_{m} \frac{\left\langle n_{m}\right\rangle-1}{2} \\
& =\frac{1}{N} \sum_{m} \frac{\bar{\mu} \sinh \left(\beta \Delta_{m}\right)}{2 \Delta_{m}\left[\exp \left(\beta \frac{U}{2}\right) \cosh \left(\beta H_{\mathrm{p}}\right)+\cosh \left(\beta \Delta_{m}\right)\right]} .
\end{aligned}
$$

Variation of Eq. (3.4) with respect to $\boldsymbol{A}$ gives the expression for the superconducting current density

$$
j_{s}=\frac{\mathrm{i} e J}{\hbar^{2} d^{2}} \sum_{m m^{\prime}}\left[\psi_{m}^{+} \psi_{m^{\prime}}^{-} \exp \left(\mathrm{i} \phi_{m m^{\prime}}\right)-\text { h.c. }\right]\left(r_{m}-r_{m^{\prime}}\right)
$$


where $d$ is a distance between the sites. It follows from (3.9) that the penetration depth of a weak field is

$$
\lambda_{\mathrm{L}}=\frac{m^{*} c^{2}}{16 \pi e^{2}|\psi|^{2} / d^{3}},
$$

where $m^{*}=\hbar^{2} /\left(2 J d^{2}\right)$. For $T=0 ;|\psi|^{2}=n(2-n) / 4$.

Equations (3.4)-(3.9) provide a complete description of the superconductivity in the model (3.1) at all temperatures within the approximation used. In the limit $U \rightarrow-\infty$, if one puts $J=2 t^{2} /|U|$, these equations reduce to those derived by Bulaevskii et al. [18] for the pseudospin model (2.1), whereas for $\boldsymbol{H}=0$ we recover the results of Ref. [19]. The functional (3.4) and Eqs. (3.7)-(3.9) are of the difference type and it makes the superconducting properties of the model analogous to the properties of the Josephson systems.

We have performed a rather extensive analysis of Eqs. (3.4)-(3.9) including thermodynamics [19] and electromagnetic properties [17] for arbitrary electron concentrations and interaction parameters. We have also studied the effects of Gaussian and critical fluctuations [19]. The phase diagram of the model is plotted in Fig. 4. Notice that for $U / J_{0}<0.5$ the ground state is superconducting (SS) for $0<n<2$, whereas for $0.5<U / J_{0}<1$ the concentration range of the stable SS phase is reduced with increasing $U$.

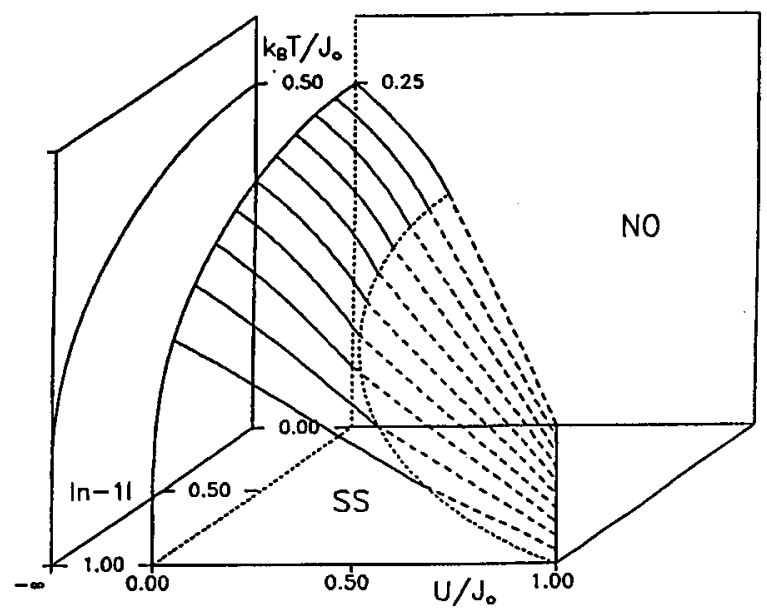

Fig. 4. Phase diagram of the model (3.1) for $W_{i j}=0$. The second and first order transitions at $T>0$ are indicated, respectively, by the solid and dashed lines. Tricritical points (TCP) are shown as a dotted line. SS is the singlet (on-site) superconducting phase, NO - the nonordered phase. $J_{0}=\sum_{j} J_{i j}$.

The properties of the system strongly depend on the ratio $U / J$, directly linked with the relative values of the pair-binding energy $E_{\mathrm{b}}=J_{0}-U$ and the pair hopping amplitude $t_{\mathrm{p}}=J$. There are two well-defined regimes of the model. The first one is the local pair limit which is realized for large pair binding energies 
$E_{\mathrm{b}} \gg t_{\mathrm{p}}$ (i.e. for $U<0$ ). In such a case, $T_{\mathrm{c}}$ is determined by the center-of-mass motion of pairs, i.e. by pair mobility, and the transition is to a state of dynamically disordered local pairs. The number of pairs remains almost unchanged by the transition. In the opposite regime, which we call the pair breaking limit and which is realized for $E_{\mathrm{b}}<t_{\mathrm{p}}$ (i.e. for substantial values of repulsive $U$ ) the transition is determined by pair breaking excitations and there are essentially no performed pairs above $T_{\mathrm{c}}$. Let us briefly conclude some of the properties of the system in both regimes.

\subsubsection{Tightly bound local pairs}

(i) The critical temperature $T_{\mathrm{c}}$ is maximum for $n=1$ (if $W_{i j}=0$ ) and it monotonically decreases with decreasing electron concentration (comp. Fig. 4).

(ii) The ratio $k_{\mathrm{B}} T_{\mathrm{c}} / E_{\mathrm{b}}$ is generally much lower than unity and it decreases with decreasing concentration and with increasing $|U|(U<0)$.

(iii) Due to large values of pair binding energy and consequently the paramagnetic critical field $\left(\mu_{\mathrm{B}} H_{\mathrm{p}}(0) \sim E_{\mathrm{b}} \gg k_{\mathrm{B}} T_{\mathrm{c}}\right)$ the major effect of the magnetic field occurs via its coupling to the orbital motion of charged local pairs. This yields much enhanced values of $H_{\mathrm{C} 2}$, proportionally reduced values of $H_{\mathrm{C} 1}$ and large penetration depth. It also provides an upward curvature of $H_{\mathrm{C} 2}$ near $T_{\mathrm{c}}$.

(iv) The pair density $n_{\mathrm{p}}=2 / N \sum_{i}\left\langle n_{i \uparrow} n_{i \downarrow}\right\rangle$ exhibits no sharp feature as the temperature is increased through $T_{\mathrm{c}}$, whereas the paramagnetic susceptibility in the normal state is strongly suppressed and exhibits a Van Vleck (or singlet-triplet) type behavior.

Generally the properties of SS state are analogous to the superfluidity of charged bosons on a lattice (equivalence of Eq. (3.1) with (2.1) for $U \rightarrow-\infty$ ).

\subsubsection{The pair breaking regime}

With increasing $U>0$ the pair binding energy and the critical temperature are reduced and finally they become almost proportional to each other, indicating that the pair-breaking effects determine $T_{\mathrm{c}}$. The physics of the system in the pair breaking regime is in many aspects qualitatively different from that in the previously discussed case.

(i) The width of the critical region $t_{\mathrm{G}}$ is strongly reduced and the GinzburgLandau (G-L) coherence length $\xi_{0}$ is enhanced.

(ii) The $T_{\mathrm{c}}$ vs. $n$ dependence is nonmonotonic (even for $W=0$ ) with the maximum of $T_{\mathrm{c}}$ shifted from $n=1$ towards lower concentrations. For small binding energies (substantial values of $U$ ) $T_{\mathrm{c}}$ drops to zero above a critical concentration which is reduced with increasing $U$ (comp. Fig. 4).

(iii) The pair density $n_{\mathrm{p}}$ exhibits a sharp break at $T_{\mathrm{c}}$. A fraction of single electrons above $T_{\mathrm{c}}$ rapidly grows with increasing $U$, and for small binding energies $n_{\mathrm{p}}\left(T \geq T_{\mathrm{c}}\right)$ becomes vanishingly small.

(iv) The paramagnetic susceptibility in the normal state is substantial and takes a Curie-type form of temperature dependence (such a form is obvious as we have considered the zero-bandwidth case).

(v) The ratio $\mu_{\mathrm{B}} H_{\mathrm{p}}(0) / k_{\mathrm{B}} T_{\mathrm{c}}$ is of an order of unity (the Clogston limit) and in contrast to the local pair regime the paramagnetic effects of magnetic field can essentially contribute to electrodynamics of the system. 
Although the two extreme limits of the model are much different as far as the physics is concerned, the evolution of thermodynamical and electromagnetic properties between them as a function of the interaction parameters is smooth (compare Figs. 4-14 in Ref. [19], where the plots of $n_{\mathrm{p}}(T), \chi(T), H_{\mathrm{c}}(0), H_{\mathrm{p}}(0), \xi_{0}$ and $t_{\mathrm{G}}$ versus $U / J_{0}$ and $n$ are presented).

Let us note that in our model the G-L coherence length $\xi_{0}$ is not directly related to the pair radius $\xi_{\mathrm{p}}$. The latter quantity depends on the single-particle hopping $t_{i j}$ and the form of pairing potential and in our model of on-site pairing with $t_{i j}=0, \xi_{\mathrm{p}}=0$. On the other hand $\xi_{0}$ is a function of $U / J_{0}$ or, in other words, a function of the binding energy $E_{\mathrm{b}}$ and the pair mobility $t_{\mathrm{p}}$ and it can become appreciable if $t_{\mathrm{p}} / E_{\mathrm{b}} \geq 1$, i.e. in the pair breaking regime. At $T=0$ the explicit expression for this quantity is

$$
\begin{aligned}
& \xi_{0}=\frac{d}{\sqrt{z}}, \quad \text { for } U<0, \\
& \xi_{0}=\frac{d}{\sqrt{z}}\left[\frac{n(2-n)}{\frac{2 U}{J_{0}}(|n-1|-1)+n(2-n)}\right]^{1 / 2}, \quad \text { for } U \geq 0 .
\end{aligned}
$$

\subsection{Superconductivity with the first order transition}

A unique feature of the model (3.1) is a possibility of the first order transition (Fig. 4) which can result in the pair-breaking regime due to a competition between the repulsive $U$ and the pair hopping $J$. Expanding Eq. (3.4) up to the 6-th order in $\left|\psi_{i}^{ \pm}\right|$and taking a continuum limit one obtains [17]

$$
\begin{aligned}
F_{0}= & F_{0}(0) \\
& +\int J d^{2}\left[\left|\left(\nabla-\frac{2 \mathrm{i} e}{\hbar c} \boldsymbol{A}\right) \psi\right|^{2}+\frac{B^{2}}{8 \pi}+a|\psi|^{2}+\frac{b}{2}|\psi|^{4}+\frac{c}{3}|\psi|^{6}\right] \mathrm{d} \boldsymbol{r},
\end{aligned}
$$

where $\boldsymbol{B}=\boldsymbol{\nabla} \times \boldsymbol{A}$ and explicit expressions for the coefficients $a, b, c$ are given in Refs. $[17,19]$.

The equilibrium value $\psi=\psi_{\mathrm{c}}$ is determined by the equations

$$
\begin{aligned}
& \frac{J d^{2}}{\hbar^{2}}\left(-\mathrm{i} \hbar \nabla-\frac{2 e}{c} \boldsymbol{A}\right)^{2} \psi+a \psi+b|\psi|^{2} \psi+c|\psi|^{4} \psi=0, \\
& j=\frac{c}{4 \pi} \nabla \times \boldsymbol{B}=\frac{\mathrm{i} e 2 J d^{2}}{\hbar}\left(\psi^{*} \nabla \psi-\psi \nabla \psi^{*}\right)+\frac{8 e^{2} d^{2}}{\hbar^{2} c}|\psi|^{2} \boldsymbol{A} .
\end{aligned}
$$

If one substitutes $m^{*}=2 m=\hbar^{2} /\left(2 J d^{2}\right)$ Eqs. (3.12-3.14) take the form of the familiar G-L equations [20], generalized by inclusion of the sixth-order term $c$. The boundary conditions remain the same as in the usual case.

As follows from the analysis of the above equations the electromagnetic properties of the system near the tricritical point $(b \geq 0)$ and in the case of the first order transition $(b<0)$ are different from those of both type I and type II superconductors. Basic differences are evident from Fig. 5. In contrast to a standard type II superconductor: (i) the line $H_{\mathrm{C} 1}$ starts at a finite field, i.e. at low fields the 

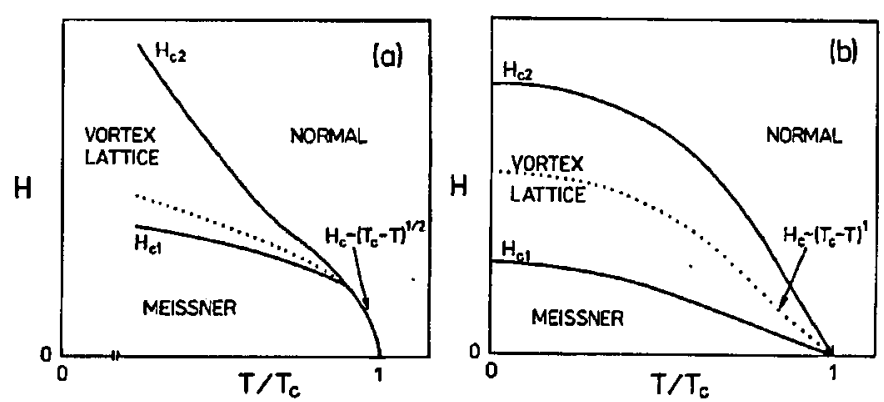

Fig. 5. (a) The $T-H$ phase diagram of the model (3.1) for the case of the first order superconducting transition at $H=0$ and $\lambda_{\mathrm{L}} / \xi_{0} \gg 1$. The transition to the normal state remains discontinuous at any $I \neq 0$. (b) The $T-H$ phase diagram of a standard type II superconductor.

mixed phase (vortex lattice) does not appear and with increasing temperature the system exhibits a direct transition from the Meissner state to the normal state; (ii) this transition is of the first order and occurs at the thermodynamical critical field

$$
H_{c}=\left[6 \pi \frac{\alpha|b|}{c}\left(T_{0}-T\right)\right]^{1 / 2},
$$

where $T_{0}$ is the critical temperature in a zero field and $\alpha=(\partial a / \partial T)_{T_{0}}$; (iii) at higher fields the structure of the phase diagram is similar to the usual case, but the transition between the mixed state and the normal state is still of the first order; (iv) near TCP the G-L parameter $\kappa$ can vary with temperature and cannot be considered as inherent constant of a particular system. Explicitly

$$
\kappa^{2}=\frac{\lambda^{2}}{\xi^{2}}=\frac{1}{2 b_{0}} \sqrt{b^{2}(T)-4 a(T) c}
$$

where $b_{0}=2 \pi e J d^{2} /\left(\hbar c^{2}\right)$,

$$
\begin{aligned}
& \xi^{2}=\frac{J d^{2}}{2 a+b|\psi|_{c}^{2}}, \\
& |\psi|_{c}^{2}=\frac{1}{2 c}\left(\sqrt{b^{2}-4 a c}-b\right)
\end{aligned}
$$

and $\lambda$ is given by Eq. (3.10). Thus, even within the G-L region $\lambda$ and $\xi$ can have different temperature dependence.

For $\kappa \gg 1$ the lower critical field $H_{\mathrm{C} 1}$ is derived as [21]

where

$$
H_{\mathrm{C} 1}(T)=H_{0} \frac{\ln \kappa}{2 \kappa}
$$

$$
H_{0}(T)=\frac{\hbar c}{2 e \xi \lambda}, \quad H_{0}\left(T_{c}\right)>0,
$$

and in such a case the intersection point of $H_{\mathrm{C} 1}$ with $H_{\mathrm{C}}$ (Fig. $5 \mathrm{a}$ ) is determined by equating the expressions (3.15) and (3.19). 


\section{Coexisting local pairs and electrons}

A model of coexisting LPs and itinerant electrons was introduced by us a few years ago $[22,23]$ and its extended versions have been analyzed and applied to real materials in a large number of more recent papers (see $[1,2]$ and references therein). It has been shown that in this type of systems a new mechanism of superconductivity can develop. It results from the intersubsystem charge exchange, both hybridization induced and a direct one, and leads to the SS state involving both types of particles. The physical properties of the model evolve with the relative concentration of LPs and electrons and they can show features which are intermediate between those of pure LP superconductors and those of BCS systems.

Up to now the studies of the model have been concentrated on the SS phase. Below we will show the relation of the model with the magnetic Kondo-lattice problem and point out a possibility of local charge moment compensation (charge Kondo effect) and the CDW orderings.

The effective Hamiltonian of local $c$-electron pairs and itinerant $a$-electrons, including only terms important for further discussion, can be written as

$$
\begin{aligned}
H= & \sum_{i j \sigma} t_{i j} a_{i \sigma}^{+} a_{j \sigma}-\mu \sum_{i \sigma} n_{i \sigma}^{a}+\left(\Delta_{0}-\mu\right) \sum_{i}\left(2 \rho_{i}^{z}+1\right) \\
& +\frac{1}{2} I \sum_{i}\left(a_{i \uparrow}^{+} a_{i \downarrow}^{+} \rho_{i}^{-}+\text {h.c. }\right)+\frac{1}{2} V \sum_{i} n_{i \sigma}^{a}\left(2 \rho_{i}^{z}+1\right),
\end{aligned}
$$

$n_{i \sigma}^{a}=a_{i \sigma}^{+} a_{\sigma}, \rho_{i}$ are charge operators of LPs defined by (2.10), $\Delta_{0}$ measures the relative position of the LP level with respect to the $a$-electron band, $I$ is the transfer component of intersubsystem charge exchange and $V$ is the density-density interaction. $\mu$ is the chemical potential depending on the total number of particles per site

$$
n=n_{\mathrm{c}}+n_{a}=\frac{1}{N} \sum_{i}\left(\left\langle 2 \rho_{i}^{z}+1\right\rangle+\sum_{\sigma}\left\langle n_{i \sigma}^{a}\right\rangle\right)
$$

Let us assume the case of alternate $(\mathrm{AB})$ lattices and $t_{i j}$ restricted to $\mathrm{nn}$ and perform the canonical "attraction-repulsion" transformation $[1,8]$

$$
\begin{aligned}
& c_{i \uparrow}^{+}=d_{i \uparrow}^{+}, \quad a_{i \uparrow}^{+}=b_{i \uparrow}^{+}, \\
& c_{i \downarrow}^{+}=\exp \left(\mathrm{i} \boldsymbol{Q} \cdot \boldsymbol{R}_{i}\right) d_{i \downarrow}, \quad a_{i \downarrow}^{+}=\exp \left(\mathrm{i} \boldsymbol{Q} \cdot \boldsymbol{R}_{i}\right) b_{i \downarrow},
\end{aligned}
$$

where $\exp \left(\mathrm{i} \boldsymbol{Q} \cdot \boldsymbol{R}_{i}\right)=+/-$ for $i \in \mathrm{A} / \mathrm{B}$.

The Hamiltonian (4.1) is thus transformed into

$$
\begin{aligned}
H= & \sum_{i \sigma} t_{i j} b_{i \sigma}^{+} b_{j \sigma}-\bar{\mu} \sum_{i}\left(2 \sigma_{i b}^{z}+1\right)+\left(\Delta_{0}-\bar{\mu}\right) \sum_{i}\left(2 S_{i}^{z}+1\right) \\
& +\frac{1}{2} I \sum_{i}\left(\sigma_{i b}^{+} S_{i}^{-}+\text {h.c. }\right)+V \sum_{i} \sigma_{i b}^{z} S_{i}^{z}+\text { const }
\end{aligned}
$$

where $\vec{\mu}=\mu-V / 4$,

$$
\sigma_{i b}^{+}=b_{i \uparrow}^{+} b_{i \downarrow}, \quad S_{i}^{+}=d_{i \uparrow}^{+} d_{i \downarrow},
$$




$$
\sigma_{i b}^{z}=\frac{1}{2}\left(n_{i \uparrow}^{b}-n_{i \downarrow}^{b}\right), \quad S_{i}^{z}=\frac{1}{2}\left(n_{i \uparrow}^{d}-n_{i \downarrow}^{d}\right),
$$

whereas Eq. (4.2) and the obvious equalities $1 / N \sum_{i}\left\langle\sigma_{i a}^{z}\right\rangle=0,\left(n_{i \uparrow}^{c}-n_{i \downarrow}^{c}\right)^{2}=0$ provide the auxiliary conditions

$$
\begin{aligned}
& \frac{n-2}{2}=\frac{1}{N} \sum_{i}\left\langle\sigma_{i b}^{z}+S_{i}^{z}\right\rangle, \\
& \frac{1}{N} \sum_{i \sigma}\left\langle n_{i \sigma}^{b}\right\rangle=1, \quad n_{i \uparrow}^{d}+n_{i \downarrow}^{d}=1 .
\end{aligned}
$$

The transformed Hamiltonian:(4.4) has the form of a magnetic Kondo-lattice model with anisotropic contact coupling and with a half-filled band of itinerant electrons, Eq. (4.7). In general case, there are two external fields: $2 \bar{\mu}$ - acting on itinerant electrons and $2\left(\bar{\mu}-\Delta_{0}\right)$ - acting on localized spins, such that total magnetization per site in $z$-direction has the fixed value $(n-2) / 2, \mathrm{Eq}$. (4.6). If $n=2$ and $\Delta_{0}=0$ one obtains $\bar{\mu}=0$ and for $I=V$ we recover the symmetric Kondo-lattice without a field.

In our studies of the anisotropic case we have used the functional integral method within a static approximation as it provides a simple description of the competition between RKKY interactions and Kondo singlet formation. Upon transformation of the obtained results into the original problem one can draw the following conclusions.

(i) For small values of $I / D$ and $V / D$, where $D=z t$, the system can exhibit the CDW, SS or SS-CDW orderings and their mutual stability is dependent on the $I / V$ ratio and the particle concentration.

(ii) CDW is stabilized by the density interaction $V$ and is favored near the half-filling of the bands. It involves spatial modulation of charge in both subsystems. With increasing $V / D$ the CDW transition temperature has a round maximum at a definite value of $V / D$ dependent on $n, \Delta_{0}, I / D$ and goes asymptotically to zero for $V / D \rightarrow \infty$.

(iii) Increasing $I / D$ reduces the charge moment of LPs. For large values of $I / D$ the charged Kondo-lattice state with a local charge moment compensation (isospin singlet [24]) can develop suppressing CDW and SS. At $T=0$ the narrow quasiparticle band appearing near $E_{\mathrm{F}}$ is split by a coherence gap $E_{\mathrm{c}}$. The $E_{\mathrm{c}}$ disappears progressively when $T$ increases and at high temperatures the systems enter the incoherent charge Kondo regime and then the logarithmic regime with the properties similar to those of a single charge Kondo impurity analyzed in Ref. [24].

\section{Acknowledgments}

The author wishes to thank R. Micnas and T. Kostyrko for stimulating discussions. The work has been supported by the Committee for Scientific Research (KBN) under the contracts 200119101 and 2P3 0205704. 


\section{References}

[1] R. Micnas, J. Ranninger, S. Robaszkiewicz, Rev. Mod. Phys. 62, 113 (1990).

[2] R. Micnas, S. Robaszkiewicz, in: Ordering Phenomena in Condensed Matter Physics, Proc. of XXVI Karpacz Winter School of Theoretical Physics, Eds. Z.M. Galasiewicz, A. Pękalski, World Scientific, Singapore 1991, p. 127.

[3] N.F. Mott, Adv. Phys. 39, 55 (1991); L.J. de Jongh, Physica C 161, 631 (1989).

[4] Y.J. Uemura, G.M. Luke, B.J. Sternlieb, J.H. Brewer, J.F. Carolan, W.N. Hardy, R. Kadono, J.R. Kempton, R.F. Kiefl, S.R. Kreitzman, P. Mulhern, T.M. Riseman, D.Ll. Williams, B.X. Yang, S. Uchida, H. Takagi, J. Gopalakrishnan, A.W. Sleight, M.A. Subramanian, C.L. Chien, M.Z. Cieplak, Gang Xiao, V.Y. Lee, B.W. Statt, C.E. Stronach, W.J. Kossler, X.H. Yu, Phys. Rev. Lett. 62, 2317 (1989); Y.J. Uemura, L.P. Le, G.M. Luke, B.J. Sternlieb, W.D. Wu, J.H. Brewer, T.M. Riseman, C.L. Seaman, H.B. Maple, M. Ishikawa, D.G. Hinks, J.D. Jorgensen, G. Saito, H. Yamochi, 66, 2665 (1991).

[5] T. Schneider, H. Keller, Phys. Rev. Lett. 69, 3374 (1992); Physica C 207, 366 (1993).

[6] T. Kato, Prog. Theor. Phys. 4, 514 (1949).

[7] M. Takahashi, J. Phys. C 10, 1289 (1977).

[8] S. Robaszkiewicz, R. Micnas, K.A. Chao, Phys. Rev. B 23, 1447 (1981); 24, 1579 (1981).

[9] R. Micnas, S. Robaszkiewicz, Phys. Rev. B 45, 9900 (1992).

[10] A.H. MacDonald, S.M. Girvin, D. Yoshioka, Phys. Rev. B 37, 9753 (1988).

[11] H.B. Callen, Phys. Rev. 130, 890 (1963); J. Adler, J. Oitmaa, J. Phys. C 12, 575 (1979).

[12] R.T. Scalettar, D.J. Scalapino, R.L. Sugar, D. Toussaint, Phys. Rev. B 39, 4711 (1989).

[13] H. Hasegawa, J. Phys., Condens. Matter 1, 9325 (1989).

[14] R.A. Bari, Phys. Rev. B 3, 2662 (1971).

[15] S. Robaszkiewicz, Acta Phys. Pol. A 55, 453 (1979).

[16] R. Micnas, S. Robaszkiewicz, K.A. Chao, Phys. Rev. B 29, 2784 (1984); Physica A 132, 504 (1985).

[17] S. Robaszkiewicz, unpublished.

[18] L.N. Bulaevskii, A.A. Sobyanin, D.I. Khomskii, Zh. Eksp. Teor. Fiz. 87, 1490 (1984).

[19] S. Robaszkiewicz, G. Pawłowski, Physica C 210, 61 (1993).

[20] L.N. Bulaevskii, V.L. Ginzburg, A.A. Sobyanin, Physica C 152, 378 (1988).

[21] I.A. Fomin, B. Lyons, J. Phys., Condens. Matter 5, 3801 (1993); I.A. Fomin, J. Phys., Condens. Matter 5, 217 (1993).

[22] J. Ranninger, S. Robaszkiewicz, Physica B 135, 468 (1985).

[23] S. Robaszkiewicz, R. Micnas, J. Ranninger, Phys. Rev. B 36, 180 (1987).

[24] A. Tarapder, P. Coleman, Phys. Rev. Lett. 66, 2814 (1991). 